\title{
Modelación morfológica del río Cauca en el tramo La Balsa-Juanchito
}

\author{
Carlos A. Ramírez*, Ricardo A. Bocanegra*\$ , María C. Sandoval** \\ * EIDENAR, Facultad de Ingeniería, Universidad del Valle, Cali, Colombia \\ **COAT, Subdirección de Gestión Ambiental, Corporación Autónoma Regional del Valle del Cauca \\ §e-mail: ricardoa@univalle.edu.co
}

(Recibido: Marzo 16 de 2006 - Aceptado: Septiembre 27 de 2006)

\begin{abstract}
Resumen
Cualquier intervención natural o antrópica en un río origina cambios en su morfología que pueden propagarse grandes distancias aguas arriba y aguas abajo del sitio intervenido, generando riesgos para las diferentes estructuras existentes, desbordamientos, etc. Predecir los cambios morfológicos en un río es posible a través de la modelación. La Corporación Autónoma Regional del Valle del Cauca, CVC, y la Universidad del Valle desarrollaron un modelo morfológico unidimensional (con base en el modelo MIKE 11) para el Río Cauca en el tramo de 110 kilómetros de longitud entre La Balsa y Juanchito. Se efectuó una primera calibración del modelo para el período 1986-2000 utilizando información suministrada por CVC y obtenida de programas de campo. Se realizó un análisis de sensibilidad que permitió identificar los parámetros que ejercen una mayor influencia en los procesos de agradación y degradación del lecho. La modelación desarrollada evidencia procesos graduales y alternados de erosión y sedimentación en el tiempo a lo largo del río debido a la variación estacional de los caudales.
\end{abstract}

Palabras clave: Río Cauca, Modelación sedimentológica, Modelación morfológica.

\section{Morphological modeling of Cauca river in the reach between La Balsa and Juanchito}

\begin{abstract}
Any anthropic or natural intervention in a river causes alterations in its morphology. These alterations can propagate to very long distances downstream and upstream, creating risks for the different structures, floodings, etc. Assessment of the morphological changes in a river is possible through mathematical modeling. Corporación Autónoma Regional del Valle del Cauca, CVC, and Universidad del Valle developed a one-dimensional mathematical model (based in model MIKE 11) for the Cauca River in the reach of $110 \mathrm{~km}$ length between La Balsa and Juanchito. A first calibration was developed for the 1986-2000 period by using information of CVC and from project field measurements. A sensibility analysis allowed the identification of parameters and variables that exert the major influences on the aggradation and degradation processes in the river. The model results indicate gradual and alternated sediment erosion processes along the river due to the seasonal variation of the discharges.
\end{abstract}

Keywords: Cauca River, Sedimentological modeling, Morphological modeling. 


\section{Introducción}

El Río Cauca es uno de los ríos más importantes de Colombia por cuanto brinda grandes beneficios a los 9 departamentos que recorre. Presenta una longitud total de $1350 \mathrm{~km}$ y una cuenca de 63300 $\mathrm{km}^{2}$. En la presente investigación se estudió el tramo comprendido entre las estaciones La Balsa y Juanchito, el cual es intervenido de manera intensa para distintos propósitos. Estas intervenciones, en conjunto con la dinámica natural del río, generan cambios en las características hidrodinámicas y morfológicas del sistema fluvial.

Predecir las respuestas del río es una tarea compleja por la gran cantidad de parámetros que intervienen en los procesos fluviales. Estas respuestas dependen en gran medida de la hidrología del río y su cuenca, la pendiente del cauce, el transporte de sedimentos y los cambios morfológicos asociados que toman lugar en el río. En muchos casos una estimación del comportamiento del río sólo puede ser alcanzada usando, en conjunto con otras herramientas, los modelos hidráulicos, los cuales pueden ser matemáticos, físicos e híbridos. Los modelos matemáticos son cada vez más usados por su gran flexibilidad y bajo costo.

La autoridad ambiental regional, CVC, y la Universidad del Valle han logrado consolidar un trabajo interdisciplinario e interinstitucional orientado a la gestión del recurso hídrico, particularmente del Río Cauca y su cuenca. Dentro de este contexto se han elaborado diversos estudios (hidrológicos, hidráulicos y de calidad de agua, entre otros) y se ha implementado un sistema de modelación matemática integral en el Río Cauca que considera aspectos hidrodinámicos, sedimentológicos, morfológicos y de calidad del agua para un tramo de aproximadamente 410 kilómetros de longitud comprendido entre las estaciones La Balsa y La Virginia. La modelación se realizó utilizando el software MIKE 11 desarrollado por el Instituto de Hidráulica de Dinamarca. En el presente artículo se presentan los resultados de los modelos sedimentológico y morfológico para el tramo La Balsa-Juanchito de aproximadamente $110 \mathrm{~km}$ de longitud.

La información disponible para desarrollar el modelo es limitada. A pesar de contar con un volumen importante de registros hidrológicos de campo, sólo se cuenta con información batimétrica del cauce del año 1986 (desde Salvajina hasta aproximadamente 10 kilómetros aguas abajo de la estación Juanchito) y del año 2000 (para todo el tramo) y la información relativa a las características y procesos sedimentológicos y morfológicos en el Río Cauca es muy reducida. Por este motivo, en la presente investigación, la calibración de los modelos sedimentológico y morfológico deben ser consideradas como una aproximación inicial a un modelo morfológico del Río Cauca.

\section{Metodología}

\subsection{Modelo matemático}

Se implementó el modelo matemático MIKE 11, que es un modelo unidimensional para la simulación de flujos, calidad del agua, transporte de sedimentos y procesos morfológicos de erosión y sedimentación en ríos, estuarios, redes de canales de riego y drenaje y otros cuerpos de agua.

MIKE 11 es un sistema de programación integrado, que conecta un número de módulos independientes a través de un sistema de menú estructurado. El módulo Hidrodinámico (HD) se constituye en el núcleo principal del sistema de modelación. Este módulo resuelve las ecuaciones de Saint Venant:

Ecuación de continuidad o de conservación de la masa de agua:

$$
\frac{\partial Q}{\partial x}+\frac{\partial A}{\partial t}=q
$$

Ecuación de conservación de la cantidad de movimiento:

$$
\frac{\partial Q}{\partial t}+\frac{\partial\left(\alpha \frac{Q^{2}}{A}\right)}{\partial x}+g A \frac{\partial h}{\partial x}+\frac{g Q|Q|}{C^{2} A R}=0
$$

donde $t(\mathrm{~s})$ es el tiempo, $x(\mathrm{~m})$ es la distancia medida a lo largo del eje del canal, $Q\left(\mathrm{~m}^{3} / \mathrm{s}\right)$ es el 
caudal, $A\left(\mathrm{~m}^{2}\right)$ es el área hidráulica, $q\left(\mathrm{~m}^{3} / \mathrm{s}\right)$ es el flujo lateral (descargas de tributarios y captaciones), $h(\mathrm{~m})$ es la altura del nivel de agua con respecto al datum o nivel de referencia, $C\left(\mathrm{~m}^{1 / 2} / \mathrm{s}\right)$ es el coeficiente de rugosidad de Chezy, $R(\mathrm{~m})$ es el radio hidráulico, $g$ es la aceleración de la gravedad y $\alpha$ es un factor de corrección debido a la distribución no uniforme de la velocidad en el término de advección o transporte.

El módulo de transporte de sedimentos no cohesivos y morfología (ST) resuelve las siguientes ecuaciones:

- Ecuación de continuidad:

$$
(1-n) \frac{\partial z}{\partial t}+\frac{\partial S}{\partial x}=0
$$

-Ecuación de Transporte de Sedimentos

$$
S=f(u, d, b, \ldots \ldots)
$$

donde $n$ es la porosidad, $z(\mathrm{~m})$ es el nivel del fondo, $S$ (ton $/ \mathrm{d}$ ) es el transporte de sedimentos, $u$ $(\mathrm{m} / \mathrm{s})$ es la velocidad, $d(\mathrm{~m})$ es el diámetro del sedimento y $b(\mathrm{~m})$ es el ancho.

\subsection{Características del río Cauca}

El río Cauca nace en el Macizo Colombiano, cerca del Páramo Sotará en el Departamento del Cauca, con ubicación $2^{\circ} 00^{\prime}$ latitud Norte y $76^{\circ} 34^{\prime}$ longitud Oeste y una elevación de $4000 \mathrm{msnm}$, y desemboca en el Brazo de Loba del río Magdalena frente al Municipio de Pinillos, en el Departamento de Bolívar a los $8^{\circ} 55^{\prime}$ latitud Norte y $74^{\circ} 29^{\prime}$ longitud Oeste, luego de atravesar gran parte del territorio nacional, encauzado entre las cordilleras Occidental y Central.

El río Cauca es un cauce aluvial caracterizado por la gran movilidad de sus meandros. El lecho, las orillas y las planicies de inundación están constituidos por materiales aluviales transportados por el mismo río. El tramo comprendido entre las estaciones La Balsa y Juanchito tiene una longitud aproximada de 110 kilómetros (ver Figura 1), presenta una sinuosidad media igual a $2.3 \mathrm{y}$ un ancho y una profundidad medios a banca llena de 100 metros y 7.5 metros respectivamente. El caudal medio en la estación La Balsa es igual a $176.7 \mathrm{~m}^{3} / \mathrm{s}$ y en la estación Juanchito es igual $258.8 \mathrm{~m}^{3} / \mathrm{s}$.

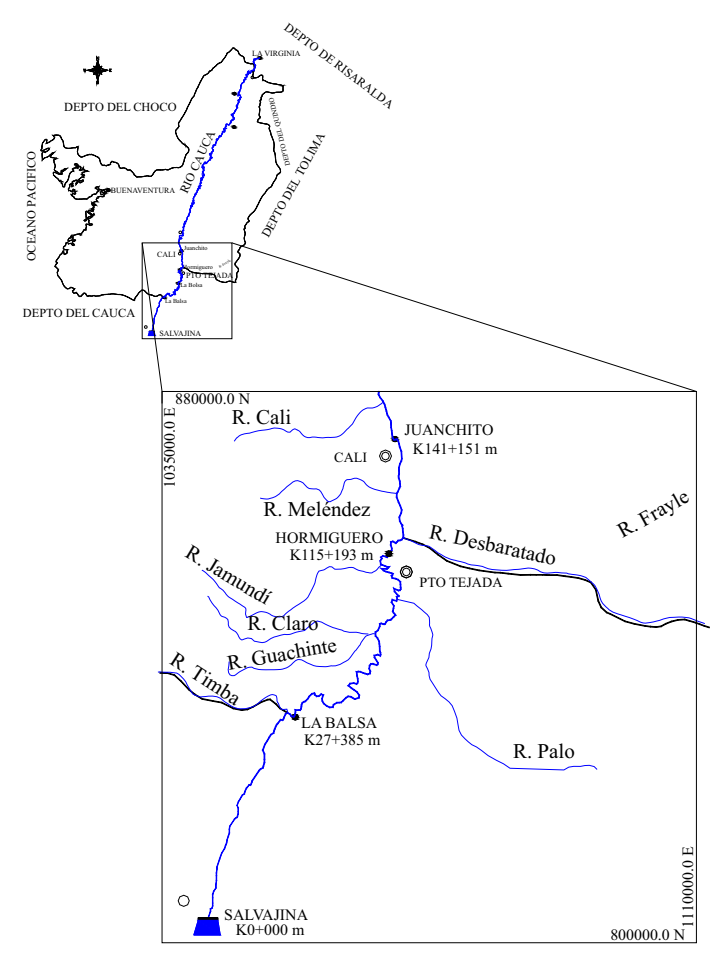

Figura 1. Tramo de estudio La Balsa-Juanchito.

En el tramo desembocan aproximadamente 20 afluentes constituidos por ríos, quebradas, canales y zanjones. Los ríos tributarios son de corta longitud, pendiente fuerte, pueden clasificarse como torrentes, con tendencia a presentar crecientes importantes de corta duración. El régimen hidrológico de estos ríos es irregular con grandes diferencias entre los caudales mínimos y máximos anuales. El régimen anual de caudales presenta dos períodos húmedos entre los meses de abril - junio y octubre - diciembre y dos períodos de estiaje entre los meses de enero - marzo y julio septiembre. Los ríos más importantes en este tramo son: Palo $\left(35.9 \mathrm{~m}^{3} / \mathrm{s}\right)$, Jamundí $\left(10.90 \mathrm{~m}^{3} / \mathrm{s}\right)$, Claro $\left(7 \mathrm{~m}^{3} / \mathrm{s}\right)$ y Desbaratado $\left(2.55 \mathrm{~m}^{3} / \mathrm{s}\right)$.

En el año 1985 entró en operación el embalse de Salvajina, localizado a aproximadamente a 170 kilómetros del nacimiento. Al comparar los registros disponibles de los períodos Pre-Salvajina y Post-Salvajina se observa que el régimen 
hidrológico del río Cauca ha experimentado variaciones: los caudales máximos se han reducido y los caudales mínimos han aumentado. Considerando únicamente los caudales promedios multianuales, estos caudales han disminuido por el efecto del embalse en promedio en un $11 \%$. Los niveles de agua promedios multianuales han descendido aproximadamente unos $0.21 \mathrm{~m}$. En la estación Juanchito el caudal máximo (promedio multianual) se redujo de $772.7 \mathrm{~m}^{3} / \mathrm{s}$ a $630 \mathrm{~m}^{3} / \mathrm{s}$, mientras que el caudal mínimo (promedio multianual) aumentó de 77.1 a $109.8 \mathrm{~m}^{3} / \mathrm{s}$ : Esta variación del régimen hidrológico es atribuible a diversos factores, tales como el efecto regulador del embalse de Salvajina, la deforestación de las cuencas tributarias, los cambios en el uso del suelo, las variaciones morfológicas, etc. De otro lado, deben tenerse en cuenta las diferencias en la extensión de los períodos de registro, por cuanto sistemáticamente para todas las estaciones hidrométricas se dispone de un mayor número de años de información hidrológica en el período Pre-Salvajina.

La variación del régimen de caudales que ha experimentado el río entre los períodos PreSalvajina y Post-Salvajina ha originado a su vez cambios en los parámetros hidráulicos y geométricos del cauce, principalmente en las estaciones La Balsa, La Bolsa y Hormiguero.

La carga total en suspensión media multianual es igual a 700000 toneladas en la estación La Balsa y a 2210000 toneladas en la estación Juanchito, con una carga de lavado que puede representar como mínimo el $77.5 \%$ de la carga total en suspensión. Después de la puesta en operación del embalse de Salvajina, la reducción en la carga total en suspensión anual promedio es aún más importante que la disminución en los caudales. En promedio, la carga total en suspensión se ha reducido en un $28 \%$ para todo el tramo Salvajina-La Virginia.

El lecho del río Cauca está conformado principalmente por sedimentos granulares. En la parte más alta del tramo estudiado del Río (aproximadamente desde Salvajina $\mathrm{K} 0+000$ hasta cerca de la desembocadura del río Claro $\mathrm{K} 66+000$ ), donde la pendiente del río es relativamente alta, predominan los materiales más gruesos, principalmente gravas, guijarros $y$ piedras; en la parte baja del río (cerca de la desembocadura del río Claro hasta la estación Juanchito K139+500), sector donde la pendiente del cauce es menor y representativa del gradiente medio del valle geográfico, dominan los sedimentos granulares finos, fundamentalmente arenas de diferentes tamaños.

Se presenta una tendencia a disminuir los tamaños de los sedimentos del fondo del río Cauca en dirección aguas abajo. Esta tendencia se ve alterada en algunos sectores a causa de las descargas de materiales más gruesos de algunos de los ríos tributarios, originando un cierto incremento en los tamaños de los sedimentos en dichos sectores, especialmente en las barras tributarias que se forman aguas abajo de la confluencia en el río Cauca.

En el sector comprendido entre la represa de Salvajina y cerca de la desembocadura del río Claro se presenta una capa superficial conformada por materiales granulares gruesos y una capa subsuperficial compuesta de materiales más finos. La capa superficial está compuesta por gravas en $69.9 \%$, arenas gruesas en $14.84 \%$, arenas medias en $10.20 \%$, arenas finas en $3.48 \%$ y limos y arcillas en $1.62 \%$. y presenta las siguientes características: $D_{10}=0.450 \mathrm{~mm}, D_{50}=6.98 \mathrm{~mm}$, $D_{90}=19.92 \mathrm{~mm}, C_{\mathrm{u}}=23.23, C_{\mathrm{g}}=1.45, \sigma_{\mathrm{d}}=6.44$, donde el $n \%$ del peso del material, es menor que $D_{n}, C_{\mathrm{u}}$ es el coeficiente de uniformidad, $C_{\mathrm{g}}$ es el coeficiente de gradación y $\sigma_{\mathrm{d}}$ es la desviación estándar. De acuerdo con los valores obtenidos para $\sigma_{\mathrm{d}}, C_{\mathrm{u}}$ y $C_{\mathrm{g}}$, el material de la capa superficial presenta una gradación extendida o no uniforme, es decir, se puede clasificar como material bien gradado. La capa subsuperficial está conformada principalmente por gravas y en menor porcentaje por arenas y tiene las siguientes características medias: $D_{10}=0.43 \mathrm{~mm}, D_{50}=6.35 \mathrm{~mm}$, $D_{90}=19.65 \mathrm{~mm}, C_{\mathrm{u}}=20.05 \mathrm{~mm}, C_{\mathrm{g}}=1.18 \mathrm{~mm}$, $\sigma_{\mathrm{d}}=5.74 \mathrm{~mm}$; la desviación típica y los coeficientes de uniformidad y gradación indican que los materiales se pueden considerar como bien gradados.

En el sector comprendido entre la desembocadura del río Claro y la estación Juanchito el lecho del río Cauca está constituido por arenas gruesas 
$(25.62 \%)$, arenas medias $(40.2 \%)$, arenas finas $(24.75 \%)$, gravas $(9.25 \%)$, limos y arcillas $(0.16 \%)$. El material del fondo del cauce en este tramo presenta, en promedio, las siguientes características: $D_{10}=0.26 \mathrm{~mm}, D_{50}=0.64 \mathrm{~mm}$, $D_{90}=2.42 \mathrm{rnm}, C_{\mathrm{u}}=3.67, C_{\mathrm{g}}=1.01, \sigma_{\mathrm{d}}=2.59$; según la desviación típica y los coeficientes de uniformidad y gradación obtenidos los sedimentos del lecho en este sector presentan una granulometría uniforme o no extendida, es decir, son mal gradados.

La explotación de materiales del fondo del río Cauca para su uso en construcción es una práctica común desde hace muchos años. En un inventario realizado por CVC en el año 1998 se encontraron alrededor de 50 dragas en el tramo Salvajina-La Virginia. Cada una de estas dragas extrae en promedio $80 \mathrm{~m}^{3} /$ día, para un total anual de 1.2 millones de $\mathrm{m}^{3}$. Esta extracción, sumada a la explotación manual que puede ascender a unos $100000 \mathrm{~m}^{3}$ anuales, está generando un impacto importante en el equilibrio del río Cauca.

\subsection{Información disponible}

Información hidrológica. Se dispone de un registro relativamente extenso de caudales en las estaciones hidrométricas ubicadas sobre el Río Cauca y 22 de sus tributarios más importantes. Se dispone de caudales diarios en todas las estaciones (la serie más larga corresponde a la estación Juanchito con registros desde 1934), curvas de calibración nivel - caudal, curvas de duración de caudales y niveles, curvas de variación estacional, entre otras. En los ríos tributarios sólo se cuenta con información en las estaciones hidrométricas; los caudales de estos ríos en las respectivas desembocaduras fueron generados utilizando el modelo hidrológico HBV (el cual es operado y aplicado desde hace varios años por la CVC).

Granulometría. La información granulométrica es bastante escasa. Sólo se dispone de la siguiente información: (i) mediciones puntuales y aisladas en los años 1976, 1977, 1978, 1981, 1985, 1987 y 1999; (ii) mediciones con un número de muestras aceptable en el año 1980; y, (iii) 96 muestras tomadas durante el muestreo sedimentológico del material de fondo del río Cauca y sus principales tributarios efectuado en el año 2003 en desarrollo de la presente investigación.

Transporte de sedimentos. Se dispone de mediciones del transporte total de sedimentos en suspensión en las estaciones hidrométricas ubicadas sobre el río Cauca y sobre algunos tributarios para un período extenso, sin embargo, la frecuencia de muestreo es relativamente baja. En desarrollo del Estudio morfológico del río Cauca (Universidad del Valle-CVC, 1986) se adelantó un estudio para establecer la carga de lavado. Adicionalmente, durante la fase I del proyecto PMC se realizó un análisis sobre la carga de lavado transportada por el río en la estación Juanchito.

El transporte o carga de fondo sólo ha sido medido durante la ejecución del Estudio Morfológico desarrollado por la CVC y la Universidad del Valle con financiación parcial de Colciencias entre los años 1980 y 1981. Las mediciones fueron realizadas en las estaciones Tablanca, Navarro, Paso de la Torre y San Francisco utilizando el equipo BTMA (Bed Transport Meter Arnhem). En los ríos tributarios no se dispone de mediciones del transporte de fondo.

Geometría del cauce del río Cauca. La batimetría del Río Cauca ha sido determinada en pocas oportunidades con diferentes niveles de detalle. La información disponible es la siguiente:

(i) Batimetría realizada en el año 1986 entre Asnazú y Puerto Isaac en desarrollo del Estudio de Navegabilidad realizado por la Flota Mercante Grancolombiana. Se levantaron 290 secciones transversales separadas en promedio cada 600 metros y ubicadas principalmente en cruces de los meandros, curvas, puntos de contracción o expansión y confluencias de los ríos tributarios.

(ii) Batimetría realizada en el año 1998 (en el desarrollo de esta investigación) entre Salvajina y la estación La Virginia. Esta campaña fue realizada entre los meses de agosto y septiembre y comprendió el levantamiento de 115 secciones transversales sobre el río Cauca y 18 secciones sobre igual número de tributarios. Las secciones transversales incluyeron el canal principal (medido a través de una ecosonda digital) y una parte de la llanura de inundación (medida con 
equipos topográficos convencionales). Previamente a la medición de las secciones transversales se construyó una red de puntos de control constituida por 57 puntos, la cual fue utilizada para determinar las elevaciones y las coordenadas de cada uno de los puntos de las secciones.

(iii) Batimetría realizada en el mes de enero de 2000 en el marco de la presente investigación entre las estaciones de Suárez y La Virginia. Esta batimetría fue realizada por medio de una ecosonda digital y un GPS siguiendo dentro del río trayectorias en zig-zag desde una orilla hasta la otra, con un ángulo promedio de $45^{\circ}$ entre cada diagonal y la dirección perpendicular en río. Se levantaron en total 3750 diagonales.

(iii) Batimetría detallada realizada en desarrollo de la presente investigación entre los meses de octubre y diciembre del año 2000. Esta campaña abarcó el tramo comprendido entre el K19+160 (cerca a la estación La Balsa) y la estación La Virginia y permitió levantar, utilizando una ecosonda digital y tecnología GPS, cerca de 9200 secciones transversales y diagonales. Para la nivelación de estas secciones se construyó una red geodésica de alta precisión (conformada por 53 puntos) a través de la cual se realizó una densificación del sistema de elevaciones y coordenadas del IGAC.

Geometría de las áreas de inundación o almacenamiento. La información existente sobre las áreas inundadas en la planicie de inundación durante los períodos de eventos de crecientes es limitada. Se dispone de planos en planta de CVC en los que se indican las áreas inundadas en los años 1949, 1950, 1966, 1970, 1971, 1974 y 1975; y de un informe de la inundación de 1988. Igualmente la CVC cuenta con vídeos de sobrevuelos de las inundaciones ocurridas en febrero y marzo de 1999. A partir de estos vídeos se determinaron las áreas inundadas sobre la cartografía desarrollada por la firma FAL Ltda. en el año 1998.

Topografía de las llanuras de inundación. Para determinar la topografía de las llanuras de inundación para los modelos hidrodinámico y morfológico se elaboró un modelo digital de elevaciones, del cual se extrajeron las secciones transversales de los canales a través de los cuales se representó la llanura de inundación. El modelo digital de elevaciones se construyó con base en la cartografía producida por FAL en 1998 y las planchetas del río Cauca y su planicie de inundación levantadas por la CVC a finales de la década de los años 70 y principios de la década de los años 80 .

\subsection{Periodo y red de modelación}

Los procesos morfológicos en un río ocurren con una menor celeridad que los procesos hidrodinámicos, por lo cual la modelación morfológica requiere de un período de tiempo suficientemente largo con el fin de lograr una calibración adecuada. Por este motivo como período de simulación se adoptó el intervalo comprendido entre enero de 1986 y diciembre de 2000 .

Para la construcción de la red de modelación se seleccionaron secciones transversales representativas de las características hidráulicas y geométricas del río, ubicadas en tramos rectos (no en curvas o cambios abruptos muy localizados, que pudiesen generar perfiles longitudinales descendentes). La geometría del río Cauca fue representada a través 50 secciones transversales separadas en promedio 2.24 kilómetros. Para efectos de calibración (comparación entre la situación real y los resultados arrojados por el modelo) se utilizaron las secciones transversales levantadas durante el período octubre - diciembre del año 2000.

Los tributarios fueron esquematizados como fuentes puntuales, con excepción de los ríos Claro, Palo y Jamundí, que fueron representados como brazos laterales a través de secciones transversales separadas cerca de 300 metros. En promedio se utilizaron 20 secciones transversales en cada río.

La planicie de inundación fue esquematizada a través de áreas de almacenamiento adicionadas a las secciones transversales después del nivel de banca llena. Dado que no se dispone de más información, estas áreas fueron obtenidas a partir de las zonas inundadas durante la creciente presentada en los meses de febrero y marzo de 
1999. Estas zonas inundadas fueron extraídas de videos obtenidos a partir de sobrevuelos y marcadas sobre cartografía IGAC actualizada por CVC en el año 1998. Para realizar las modelación se adoptó un intervalo de tiempo $\Delta t$ de 4 minutos y espaciamientos máximos $\Delta x$ de $2000 \mathrm{~m}$ para el río Cauca y $\Delta x$ de $200 \mathrm{~m}$ para los ríos tributarios. Estos valores garantizaron la estabilidad numérica del modelo (establecida a través del número de Courant para los procesos morfológicos, la condición de velocidad y los resultados del modelo) y generaron tiempos computacionales razonables.

\subsection{Fronteras del modelo}

Frontera aguas arriba. La frontera superior de la zona a modelar se localizó en la estación La Balsa. Como información hidrológica de entrada se introdujo la serie de caudales diarios para el período enero de 1986-diciembre de 2000. El transporte de sedimentos en suspensión y de fondo fue calculado empleando las ecuaciones que se presentan en la Tabla 1. Estas ecuaciones fueron determinadas durante el estudio de caracterización del río Cauca (Universidad del Valle-CVC, 2000).

Fronteras internas. Como fronteras internas se definieron 8 ríos tributarios ( 3 de los cuales fueron representados como brazos laterales), 4 extracciones de agua y 2 descargas contaminantes. La información de entrada estuvo constituida por la serie de caudales diarios generados por medio de la aplicación del modelo hidrológico HBV. Para la determinación de los transportes de sedimentos (suspensión y fondo) se emplearon las expresiones halladas durante el estudio de caracterización de los ríos tributarios al Río Cauca (Universidad del Valle-CVC, 2001). Estas ecuaciones se presentan en la Tabla 1.

Tabla 1 Expresiones empleadas para calcular el transporte de sedimentos en los ríos tributarios del río Cauca.

\begin{tabular}{llc}
\hline \multicolumn{1}{c}{ Río } & $\begin{array}{c}\text { Transporte en } \\
\text { suspensión } \\
\left.S_{\mathrm{s}} \text { (ton } / \mathrm{d}\right)\end{array}$ & $\begin{array}{c}\text { Transporte de fondo } \\
\left.S_{\mathrm{b}} \text { (ton } / \mathrm{d}\right)\end{array}$ \\
\hline Cauca & $S_{s}=0.043 Q^{1.601}$ & $S_{b}=0.028 Q^{1.288}$ \\
Palo & $S_{s}=0.037 Q^{2.329}$ & $S_{b}=0.015 Q^{2.329}$ \\
Otros afluentes & $S_{s}=0.102 Q^{1.826}$ & $S_{b}=0.041 Q^{1.826}$ \\
\hline
\end{tabular}

Frontera aguas abajo. Se localizó en la estación Juanchito. Como condición de frontera se introdujo la curva de calibración nivel-caudal.

\subsection{Calibración y verificación del modelo}

Los procesos hidrodinámicos, sedimentológicos y morfológicos en un cauce aluvial son altamente complejos debido a la interacción de múltiples parámetros (muchos de estos eminentemente empíricos) y variables. Debido a esto, predecir los cambios morfológicos en un río es una tarea bastante ardua a través de la cual sólo es posible alcanzar una aproximación a los cambios que pueden presentarse en los niveles del lecho.

El proceso seguido para la calibración del modelo sedimentológico y morfológico fue el siguiente: (i) análisis de la representatividad de la información hidrológica, geométrica y sedimentológica incluida en la modelación, (ii) modelación hidrodinámica, (iii) selección del predictor utilizado para calcular el transporte de sedimentos, (iv) selección del modelo de actualización de fondo más adecuado, (v) modelación sedimentológica (selección de los factores de ajuste de los transportes de sedimentos calculados a los transportes de sedimentos medidos), (vi) modelación morfológica (determinación de los factores de ajuste de los transportes de sedimentos calculados de acuerdo con los cambios morfológicos observados).

\subsection{Modelación hidrodinámica}

La calibración del modelo hidrodinámico se efectuó para el período de verano julio septiembre de 1998 (correspondiente a caudales medios y bajos) y la verificación para el período de invierno octubre-diciembre de 1998 (correspondiente a caudales altos). En la frontera aguas arriba se introdujo la serie de caudales horarios y en la frontera aguas abajo se introdujo la curva de calibración nivel-caudal. Se adoptó un espaciamiento máximo $\Delta x$ de $1000 \mathrm{~m}$ para el río Cauca y de $200 \mathrm{~m}$ para los ríos tributarios y un intervalo de tiempo $\Delta t$ de $2 \mathrm{~min}$, valores con los cuales se logró una representación adecuada de los procesos hidrodinámicos que se presentan en el río Cauca y sus tributarios. Además, los tiempos computacionales resultaron razonables y se garantizó la estabilidad del modelo. 
La rugosidad constituyó el principal parámetro de calibración durante la modelación hidrodinámica. Los coeficientes de rugosidad de Strickler (inverso de Manning) que permitieron alcanzar los mejores ajustes se encuentran entre 30 y 35 $\mathrm{m}^{1 / 3} / \mathrm{s}$. En ocasiones fue necesario revisar y ajustar las secciones transversales introducidas al modelo, procurando una mayor representatividad de las características morfológicas del cauce.

Los niveles de la superficie libre del agua fueron adecuadamente reproducidos por el modelo, con una aproximación que se encuentra dentro del rango de precisión considerado aceptable (entre 15 y 50 centímetros) para los modelos matemáticos, de acuerdo con Cunge et al. (1980). El modelo obtenido también reprodujo adecuadamente la variación de los caudales y las velocidades en el río Cauca.

\subsection{Modelación sedimentológica y morfológica}

Para el cálculo del transporte de sedimentos en el modelo matemático del río Cauca finalmente se seleccionó la expresión planteada por Van Rijn por cuanto: (i) se obtuvieron resultados aceptables al implementar esta metodología durante el estudio de caracterización del río Cauca (Universidad del Valle-CVC, 2000); (ii) el análisis numérico y la calibración de los parámetros que intervienen en el transporte de sedimentos es uno de los más completos (Maza-Alvarez \& Garcia-Flores, 1996); y, (iii) las simulaciones realizadas empleando todas las formulaciones disponibles en el modelo MIKE 11 indicaron que la mejor representación de las características del Río Cauca se alcanzaba al utilizar este predictor.

Dadas las características de los procesos de erosión y sedimentación en el río Cauca, se seleccionó un modelo de actualización de los niveles del lecho en el cual en promedio toda la sección transversal del cauce principal se agrada o degrada uniformemente.

En el proceso de calibración del modelo morfológico inicialmente se ajustó el transporte de sedimentos calculado por el modelo al transporte de sedimentos medido en el año 1998 en las estaciones hidrométricas ubicadas sobre el río Cauca (modelación sedimentológica). Para esto se modificaron en varias oportunidades los factores de calibración disponibles en el modelo para ajuste de la carga en suspensión y la carga de fondo. Los factores que permitieron alcanzar un ajuste razonable entre el transporte calculado y el transporte medido en campo variaron entre $0.03 \mathrm{y}$ 0.16 .

Utilizando estos factores posteriormente se simuló el período enero de 1986-diciembre de 2000 (modelación morfológica). Los resultados alcanzados mostraron tasas de erosión y sedimentación demasiado altas comparadas con las tasas y cambios que suelen presentarse en los niveles de fondo del río Cauca. Por esto, fue necesario realizar ajustes a los parámetros de calibración del modelo, así como revisar y ajustar la esquematización física del cauce, especialmente en cuanto a las características granulométricas del material del lecho.

Cuando los resultados del modelo indicaron variaciones demasiado grandes en los niveles del lecho en un tramo determinado se procedió a revisar las granulometrías introducidas para dicho tramo, encontrándose en algunos casos granulometrías muy finas comparadas con las granulometrías de otros sectores cercanos. Esto obligó a descartar las granulometrías muy finas adoptadas inicialmente en la esquematización y a sustituirlas por distribuciones de tamaños de sedimentos del material del lecho de un tramo de mayor longitud. Una vez efectuada esta modificación en la esquematización de las características del material del lecho, se obtuvieron tasas de erosión y sedimentación más representativas de las condiciones morfológicas predominantes en el río.

Adicionalmente, para lograr un mejor ajuste de los resultados del modelo a las condiciones morfológicas reales del río Cauca se definieron factores de calibración del transporte de sedimentos en secciones intermedias entre estaciones hidrométricas. Se ensayaron diferentes valores de estos factores en distintos sectores hasta alcanzar un adecuado ajuste entre los niveles de fondo calculados y medidos. 

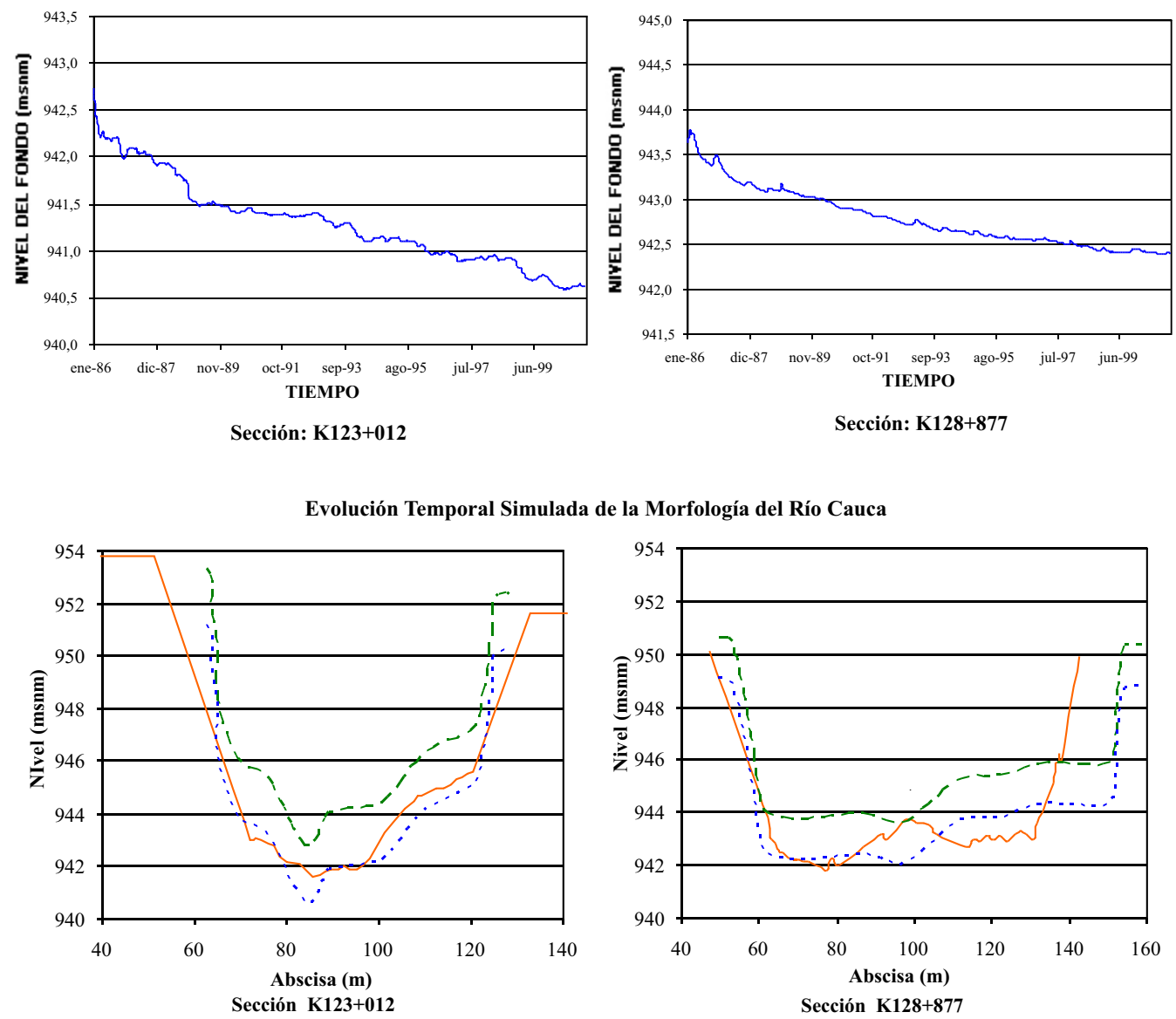

Sección Transversal Medida vs Sección Transversal Calculada

Figura 2. Calibración del modelo morfológico (abscisa K 123+012, periodo 1986 -2000).

Tabla 2. Factores de calibración adoptados en la modelación morfológica.

\begin{tabular}{l|r|r|r|r|r|r|r|r|r|r|r|r|r}
\hline Río & \multicolumn{4}{c|}{ Cauca } & \multicolumn{3}{c|}{ Palo } & \multicolumn{3}{c|}{ Claro } & \multicolumn{1}{c}{ Jamundí } \\
\hline Abscisa (km) & 27.3 & 79.9 & 87.1 & 115.2 & 128.9 & 141.2 & 0.0 & 7.6 & 0.0 & 3.3 & 5.1 & 0.0 & 6.5 \\
Factores de Calibración & 0.05 & 0.03 & 0.01 & 0.04 & 0.15 & 0.07 & 0.03 & 0.03 & 0.02 & 0.02 & 0.60 & 0.002 & 0.002 \\
\hline
\end{tabular}

\section{Resultados y discusión}

Los valores de los factores de calibración finalmente adoptados se presentan en la Tabla 2. Los resultados alcanzados en la calibración del modelo morfológico en las secciones ubicadas en las abscisas K $123+012$ y K $128+877$ se presentan en la Figura 2.

\subsection{Análisis de sensibilidad}

Con el fin de establecer o identificar los parámetros que ejercen una mayor influencia en las respuestas del modelo morfológico, se realizó un análisis de sensibilidad de los diferentes parámetros de entrada del modelo (geometría del cauce y transporte de sedimentos principalmente). Dadas las fuertes restricciones en la información disponible, este análisis reviste especial importancia ya que permite establecer los factores que deben ser analizados cuidadosamente. Los principales resultados derivados de este análisis son los siguientes:

- La composición granulométrica del material de fondo ejerce una fuerte influencia en la evolución morfológica del cauce. Las simulaciones realizadas indicaron que pequeñas variaciones en 


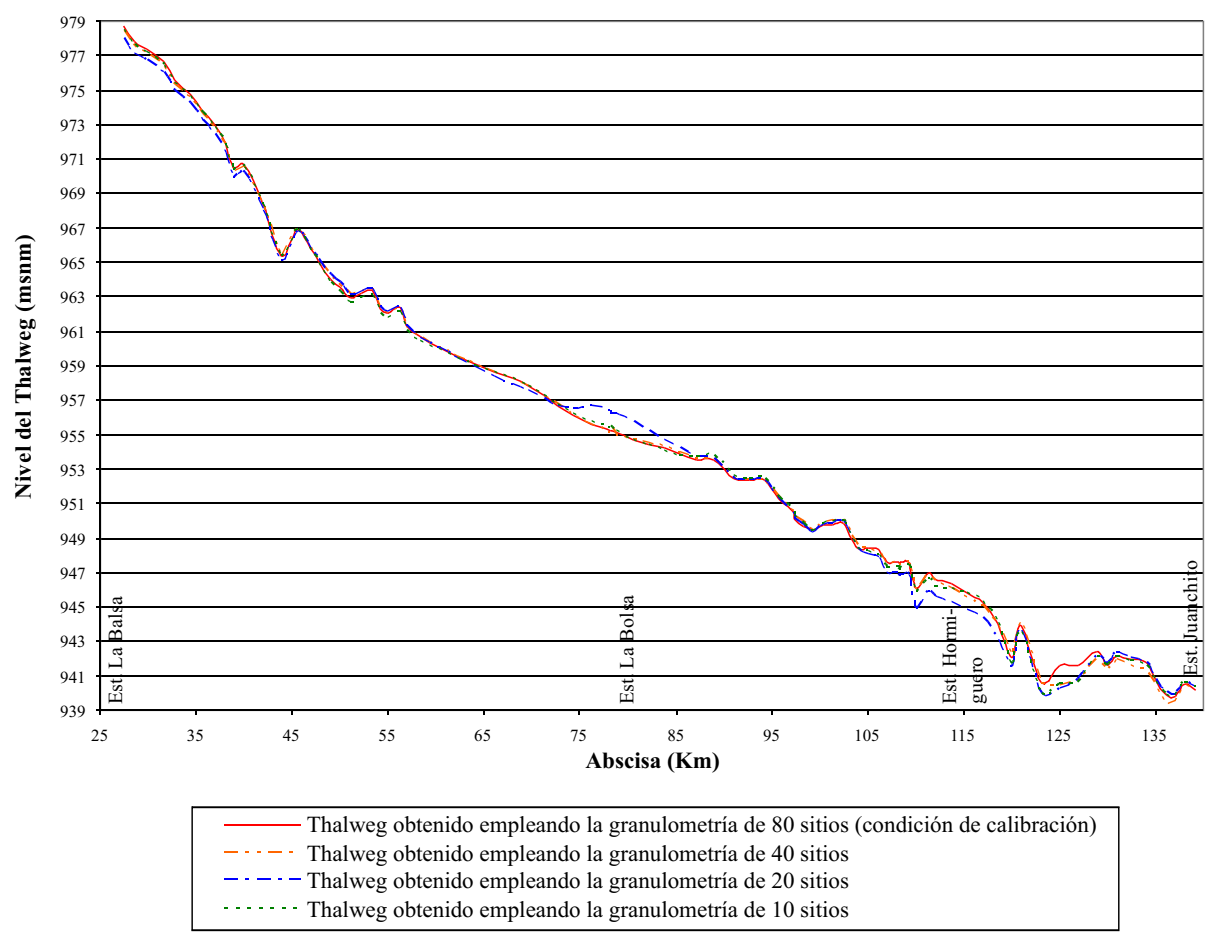

Figura 3. Influencia del grado de representación de la granulometría del material del fondo en la variación del thalweg del rio Cauca (periodo simulado: 1986 - 2000).

la granulometría del material del fondo dan lugar a variaciones importantes en la evolución morfológica del cauce del río (ver Figura 3).

- En la Figura 4 se presenta la influencia de la geometría del cauce principal en la evolución morfológica del cauce. Se observa como la representación o esquematización geométrica que se realice del sistema a modelar ejerce una influencia importante en los resultados del modelo. Secciones transversales que describan adecuadamente la geometría del canal modelado conducirán a una adecuada calibración, en tanto que secciones transversales no representativas del canal conducirán a resultados errados.

- El transporte de sedimentos en el río Cauca ejerce una fuerte influencia en los procesos morfológicos del cauce. A mayor transporte de sedimentos en el río más severos son los cambios morfológicos que experimenta su cauce. En la Figura 5 se presenta la influencia del transporte de sedimentos del río Cauca en la evolución temporal del thalweg en la abscisa K 128+877.
-El transporte de sedimentos de los ríos tributarios presenta una baja influencia en los niveles del lecho del río Cauca. A pesar de que se realizaron simulaciones empleando un rango relativamente amplio de transportes en los ríos tributarios, no se apreciaron variaciones importantes en los niveles del lecho (ver Figura 6). Esto podría explicarse como consecuencia de la cantidad relativamente baja de sedimentos que transportan los tributarios con relación al río Cauca. Sin embargo, en caso de presentarse crecientes en los ríos tributarios superiores a las ocurridas durante el período de simulación (1986-2000), los aportes de cargas de sedimentos al río Cauca pueden incrementarse ostensiblemente, lo cual podría originar fuertes cambios en los niveles del lecho del río Cauca.

- La carga de sedimentos en la frontera aguas arriba ejerce una influencia en los niveles de fondo que disminuye en dirección aguas abajo (ver Figura 7). Por ejemplo, en la sección $2(\mathrm{~K} 87+100)$, es decir, $60 \mathrm{~km}$ aguas abajo de la estación La Balsa, el cambio en el nivel del thalweg es mínimo. En contraste, en la sección $1(\mathrm{~K} 46+300)$, ubicada unos $19 \mathrm{~km}$ aguas abajo de la estación La Balsa, el lecho puede experimentar variaciones de 
Ingeniería y Competitividad, Volumen 8, No. 2, p. 80 - 93 (2006)

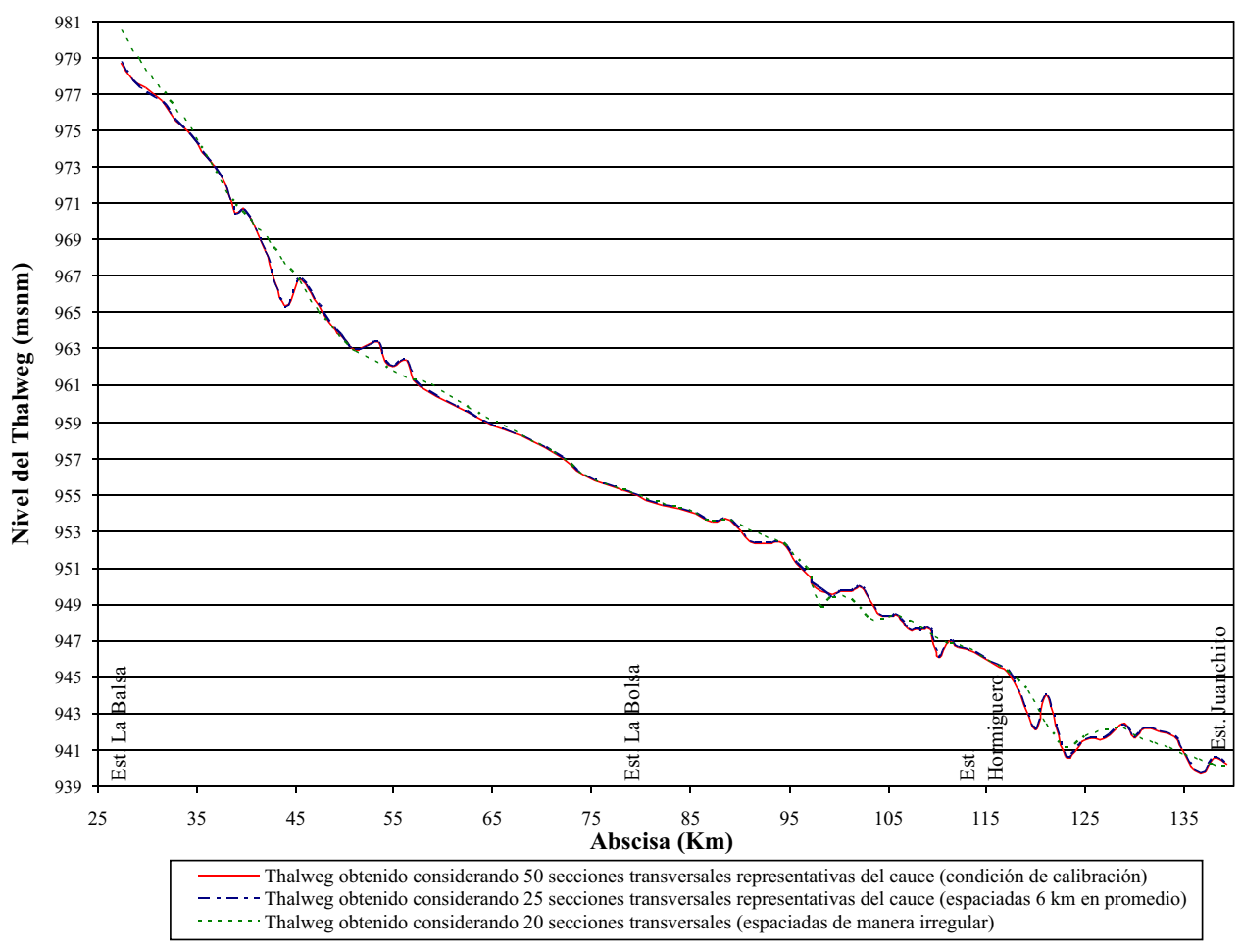

Figura 4. Influencia del grado de representación de la batimetría del cauce principal en la variación del thalweg del rio Cauca (periodo simulado: 1986-2000).

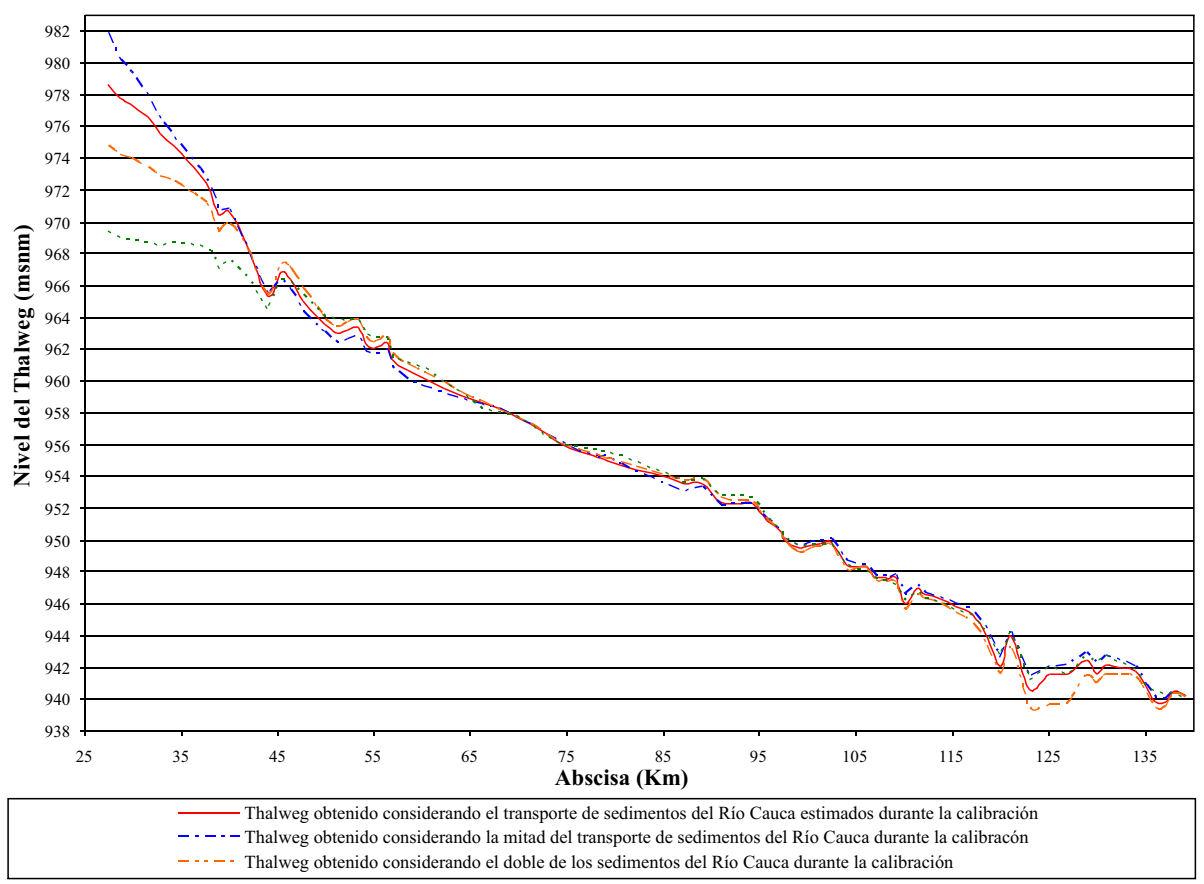

Figura 5. Influencia del transporte de sedimentos en el rio Cauca en la variación del thalweg del río (periodo simulado: 1986-2000). 


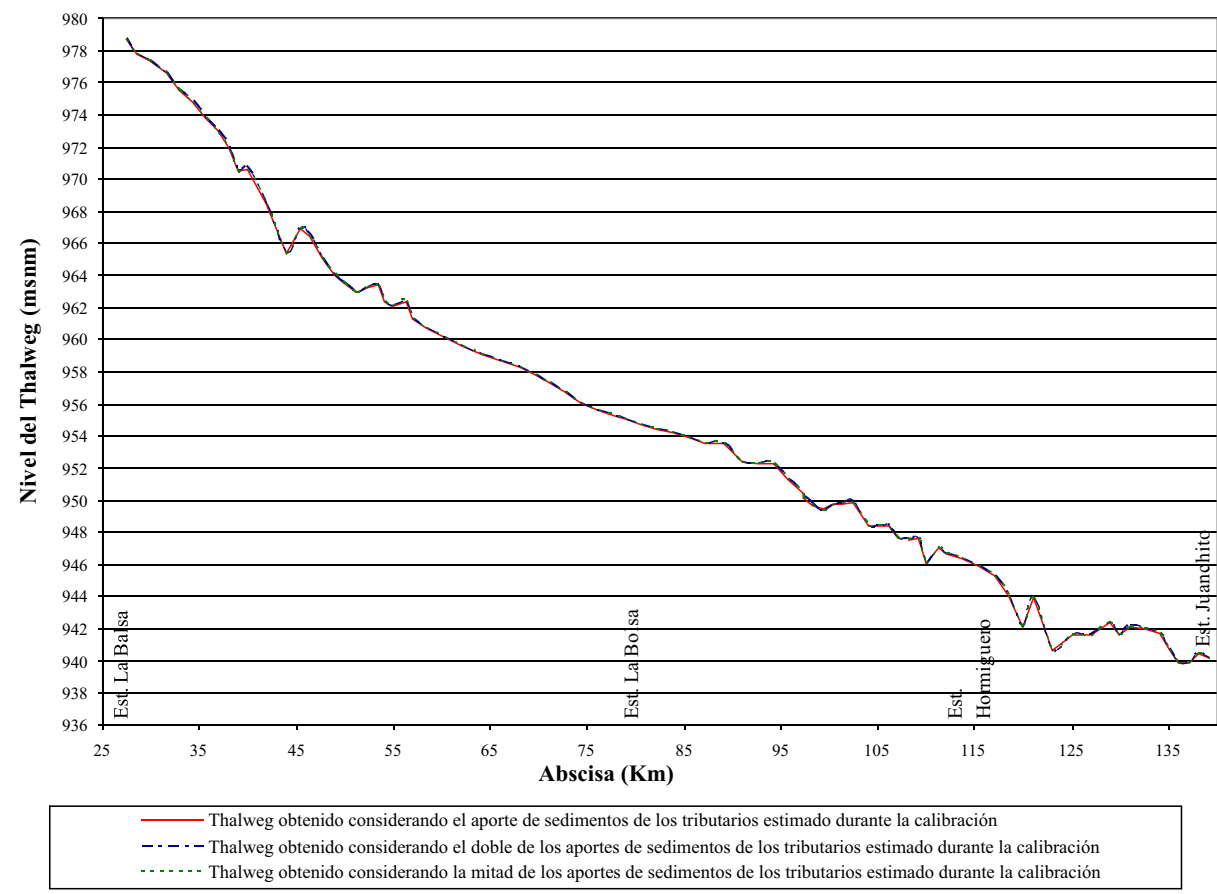

Figura 6. Influencia del transporte de sedimentos de los ríos tributarios en la variación del thalweg del rio Cauca (periodo simulado: 1986-2000).

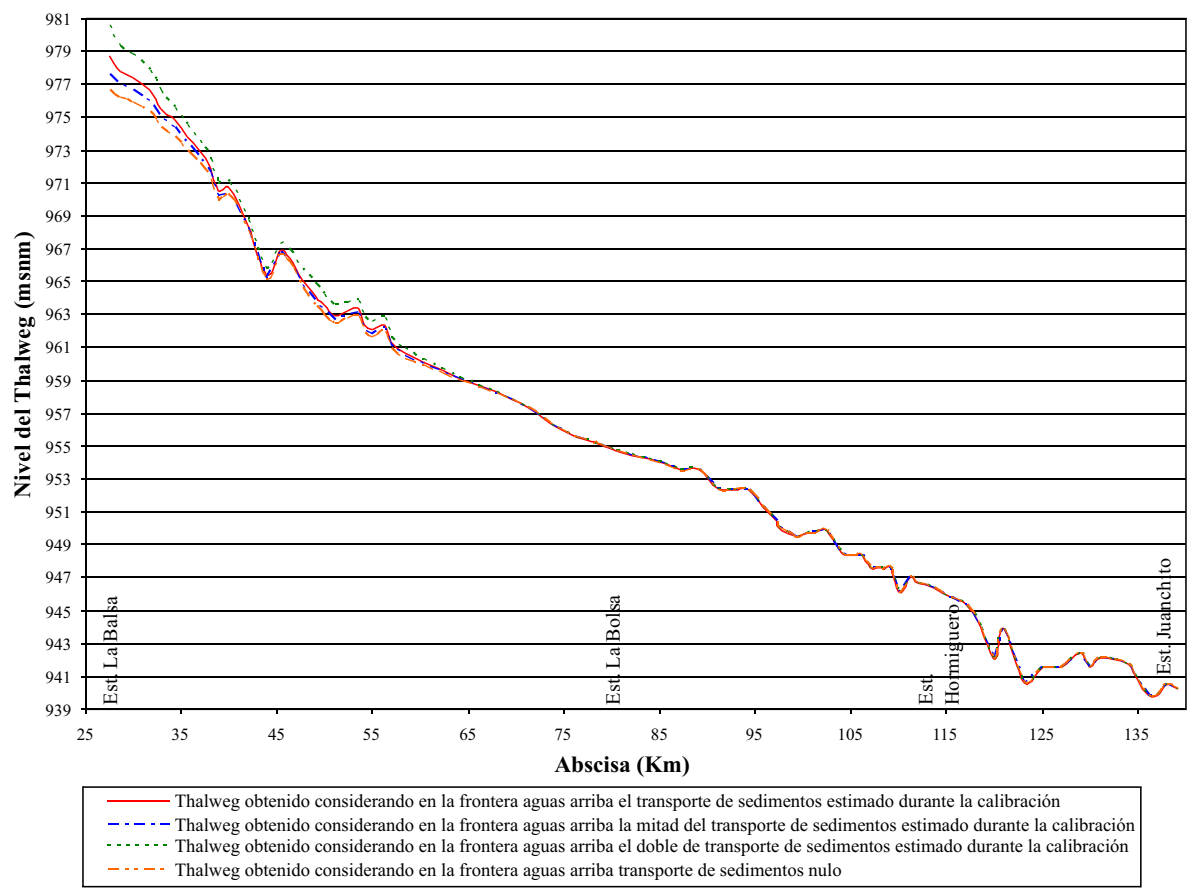

Figura 7. Influencia de la magnitud del transporte de sedimentos en la frontera aguas arriba en la variación del thalweg del río Cauca (periodo simulado: 1986-2000). 
alguna importancia (hasta de unos $0.80 \mathrm{~m}$ ) para el rango de transportes de sedimentos evaluados en la frontera (estación La Balsa).

\subsection{Análisis de resultados}

No obstante las limitaciones impuestas en la calibración del modelo morfológico debido a la escasez de información de campo disponible, es posible indicar algunos aspectos importantes con relación a los parámetros de calibración y las variaciones en los niveles del lecho del río (procesos morfológicos) como resultado de las diferentes simulaciones efectuadas.

- Como es de esperarse en un río aluvial, en el río Cauca los procesos de agradación y degradación del lecho se alternan de manera continua a lo largo del río (ver Figuras 2 a 7). Los procesos morfológicos ocurren de manera gradual, tanto temporal como espacialmente, es decir, no ocurren variaciones drásticas en los niveles del lecho en períodos cortos de tiempo (la regulación de caudales debido a la operación del embalse de Salvajina podría explicar en parte este comportamiento).

- El transporte de sedimentos en el río Cauca se incrementa en dirección aguas abajo, lo cual se ajusta al comportamiento de los ríos aluviales, en los que la capacidad de transporte del cauce aumenta en la medida en que se incrementan el caudal y la sección transversal del cauce.

- Los cambios en los niveles del fondo del cauce en unos sectores son más importantes que en otros (ver Figuras 2 a 7), lo cual está asociado a las características físicas y geométricas de cada sector y a las diferentes intervenciones antrópicas a las que viene siendo sometido el río (como por ejemplo, la intensa extracción de materiales de arrastre).

- Los principales parámetros de calibración sedimentológica y morfológica fueron los factores de ajuste de las cargas en suspensión y de fondo. Los factores finalmente hallados durante la calibración oscilaron entre 0.01 y 0.15 . Estos valores bajos sugieren, en principio, que el transporte de sedimentos en el río Cauca es inferior al transporte calculado por medio de la ecuación de Van Rijn.

- Los mayores cambios en los niveles de fondo del río Cauca se presentan durante los primeros años del período simulado, lo cual podría ser la respuesta del río a los cambios en los regímenes hidrológico y sedimentológico impuestos por la operación del embalse de Salvajina (ver Figura 2).

\section{Conclusiones}

Se construyó una herramienta que, una vez optimizada, permitirá obtener una aproximación a los cambios morfológicos del río Cauca ante diversas intervenciones humanas o antrópicas. Sin embargo, las calibraciones sedimentológica y morfológica aquí logradas deben considerarse una aproximación inicial a un modelo morfológico del río Cauca debido a las limitaciones en la información disponible (a pesar de contar con un volumen importante de registros hidrológicos, la información relativa a las características y procesos sedimentológicos y morfológicos en el río Cauca es muy reducida) y al alto grado de complejidad de los procesos hidrodinámicos, sedimentológicos y morfológicos que se presentan en un cauce aluvial.

La modelación desarrollada permitió observar que, tal como sucede en los ríos aluviales, en el río Cauca los procesos de agradación y degradación se alternan en el tiempo a lo largo del tramo modelado debido a la variación estacional de los caudales. Además, estos cambios ocurren de manera gradual tanto en el tiempo como en el espacio.

La variabilidad espacial de la composición granulométrica del material del fondo y el transporte de sedimentos del río Cauca ejercen una fuerte influencia en la evolución morfológica de su cauce. En contraste, los niveles del lecho del río Cauca se ven poco influenciados por el aporte de sedimentos de los ríos tributarios, lo cual podría explicarse por los relativamente bajos volúmenes de sedimentos que descargan estos ríos en relación con los transportados por el río Cauca.

Para optimizar los modelos sedimentológicos y morfológicos se deben adelantar monitoreos permanentes del transporte de sedimentos en el río 
Cauca (incluyendo la medición del transporte de fondo), realizar batimetrías periódicas del cauce y, en general, ampliar el conocimiento que se tiene de la cuenca del río Cauca.

\section{Referencias bibliográficas}

Cunge, J. A., Holly, F. M., \& Verwey, A. (1980). Practical aspects of computational river hydraulics, London: Pitman Publishing Limited.

Maza-Alvarez, J.A., \& Garcia-Flores, M. (1996). Transporte de sedimentos. Manual de ingeniería de ríos. (Cap. 10). Series del Instituto de Ingeniería, UNAM, México .
Universidad del Valle-CVC.(2000). Caracterización del río Cauca tramo SalvajinaLa Virginia, Cali, Colombia.

Universidad del Valle-CVC.(2001). Caracterización de los ríos tributarios del río Cauca tramo Salvajina-La Virginia, Cali, Colombia. 